\title{
Norms Of Hankel-Hessenberg and Toeplitz-Hessenberg Matrices Involving Pell and Pell-Lucas Numbers
}

\section{Hasan GÖKBAS}

S emsi Tebrizi Girl Anatolian Religious Vocational High School, Konya, Turkey

\section{ABSTRACT}

We derive some sum formulas for the squares of Pell and Pell-Lucas numbers. We construct Hankel-Hessenberg and Toeplitz-Hessenberg matrices whose entries in the first column are $H H_{P}=\left(a_{i j}\right), a_{i j}=P_{i-j} ; H H_{Q}=\left(a_{i j}\right), a_{i j}=Q_{i-j}$ and $T H_{P}=\left(a_{i j}\right), a_{i j}=P_{i-j+1} ; T H_{Q}=\left(a_{i j}\right), a_{i j}=Q_{i-j+1}$, respectively where $P_{n}$ and $Q_{n}$ denote the usual Pell and Pell-Lucas numbers. Then, we found upper and lower bounds for spectral norm of these matrices.

Keywords: Euclidean norm, Spectral norm, Toeplitz matrix, Hankel matrix, Hessenberg matrix, Pell numbers, PellLucas numbers.

Mathematic subjectClassification:15A60,15A36,11B99.

\section{Introduction}

Special matrices is a widely studied subject in matrix analysis. Especially special matrices whose entries are well known number sequences have become a very interesting research subject in recent years and many authors have obtained some good results in this area. For example, the norms of Toeplitz, Hankel and Circulant matrices involving Fibonacci, Lucas, Pell and Pell-Lucas numbers were investigated in [1, 2, 5, 6, 7]. In this study, We derive some sum formulas for the squares of Pell and Pell-Lucas numbers. We construct Hankel-Hessenberg and Toeplitz-Hessenberg matrices involving Pell and Pell-Lucas numbers.

The Pell and Pell-Lucas sequences $P_{n}$ and $Q_{n}$ are defined by the recurrence relations

$$
P_{0}=0, P_{1}=1, P_{n}=2 P_{n-1}+P_{n-2} \quad \text { for } \quad n \geq 2
$$

and

$$
Q_{0}=2, Q_{1}=2, Q_{n}=2 Q_{n-1}+Q_{n-2} \quad \text { for } \quad n \geq 2 \text {. }
$$

\begin{tabular}{|c|c|c|c|c|c|c|c|}
\hline$n$ & 0 & 1 & 2 & 3 & 4 & 5 & 6 \\
\hline$P_{n}$ & 10 & 1 & 2 & 5 & 12 & 29 & 70 \\
\hline$Q_{n}$ & 2 & 2 & 6 & 14 & 34 & 11 & 82 \\
\hline
\end{tabular}

If start from $n=0$, then the Pell and Pell-Lucas sequence are given by

The following sum formulas the Pell and Pell-Lucas numbers are well known $[8,9]$ :

$$
\begin{gathered}
\sum_{k=1}^{n-1} P_{k}^{2}=\frac{P_{n} P_{n-1}}{2} \\
\sum_{k=1}^{n-1} Q_{k}^{2}=\frac{Q_{2 n-1}+2(-1)^{n}-4}{2}
\end{gathered}
$$




$$
\begin{gathered}
\sum_{k=1}^{n-1} P_{k} P_{k+1}=\frac{P_{2 n+1}-2 P_{n+1} P_{n}-1}{4} \\
\sum_{k=1}^{n-1} Q_{2 k+1}=\frac{Q_{2 n}-6}{2} \\
Q_{n}^{2}-8 P_{n}^{2}=4(-1)^{n}
\end{gathered}
$$

A matrix $H H$ is a Hankel-Hessenberg matrix if it is of the form

$$
H H=\left[\begin{array}{cccc}
a_{0} & a_{1} & \cdots & a_{n-1} \\
a_{1} & & & a_{n} \\
\vdots & & & \\
a_{n-1} & a_{n} & &
\end{array}\right]
$$

where $a_{n} \neq 0$ and $a_{k} \neq 0$ for at least one $k>0$.

A matrix $T H$ is a Toeplitz-Hessenberg matrix if it is of the form

$$
T H=\left[\begin{array}{cccc}
a_{1} & a_{0} & & \\
a_{2} & \ddots & \ddots & \\
\vdots & \ddots & \ddots & a_{0} \\
a_{n} & \cdots & a_{2} & a_{1}
\end{array}\right]
$$

where $a_{0} \neq 0$ and $a_{k} \neq 0$ for at least one $k>0$ [3].

The Euclidean norm of the matrix $A$ is defined as

$$
\|A\|_{E}=\left(\sum_{i, j=1}^{n}\left|a_{i j}\right|^{2}\right)^{1 / 2} .
$$

The spectral norm of the matrix $A$ is

$$
\|A\|_{2}=\sqrt{\max _{1 \leq i \leq n}\left|\lambda_{i}\right|}
$$

where the numbers $\lambda_{i}$ are the eigenvalues of matrix $A^{*} A$. The matrix $A^{*}$ is the conjugate transpose of the matrix $A$. The following inequality holds,

$$
\frac{1}{\sqrt{n}}\|A\|_{E} \leq\|A\|_{2} \leq\|A\|_{E}
$$

For the matrices $A=\left(a_{i j}\right)_{m \times n}$ and $B=\left(b_{i j}\right)_{m \times n}$ the Hadamard Product of these matrices is defined as

$$
B \circ C=\left(a_{i j} b_{i j}\right)_{m \times n} .
$$


Define the maximum column lenght norm $c_{1}$, and the maximum row lenght norm $r_{1}$ of any matrix $\mathrm{A}$ by

$$
r_{1}(A)=\max _{i} \sqrt{\sum_{j}\left|a_{i j}\right|^{2}}
$$

and

$$
c_{1}(A)=\max _{j} \sqrt{\sum_{i}\left|a_{i j}\right|^{2}}
$$

respectively. Let $A, B$ and $C$ be $m \times n$ matrices. If $A=B \circ C$ then

$$
\|A\|_{2} \leq r_{1}(B) c_{1}(C)[4] \text {. }
$$

\section{Main Result}

Lemma 1 If $P_{n}$ and $Q_{n}$ are nth Pell and Pell-Lucas numbers, we have

$$
\sum_{k=1}^{n} k P_{k}^{2}=\frac{(4 n+2) P_{n+1} P_{n}-P_{2 n+1}+1}{8}
$$

and

$$
\sum_{k=1}^{n} k Q_{k}^{2}=\left\{\begin{array}{l}
\frac{2 n Q_{2 n+1}-Q_{2 n}-4 n-2}{4}, \\
\frac{2 n Q_{2 n+1}-Q_{2 n}+4 n+2}{4}, \quad \text { if } n \text { is odd }
\end{array}\right.
$$

Proof. Let $A_{n}=\sum_{k=1}^{n} P_{k}^{2}=\frac{P_{n+1} P_{n}}{2}$, then

$$
\begin{aligned}
& \sum_{k=1}^{n} k P_{k}^{2}=P_{1}^{2}+2 P_{2}^{2}+3 P_{3}^{2}+\ldots+n P_{n}^{2} \\
& =\sum_{k=1}^{n} P_{k}^{2}+ \\
= & A_{n}+\left(A_{n}-A_{1}\right)+\left(A_{n}-A_{2}\right)+\ldots+\left(A_{n}-A_{n-1}\right) \\
= & n A_{n}-\sum_{i=1}^{n-1} A_{i}=n\left(\frac{P_{n+1} P_{n}}{2}\right)-\sum_{i=1}^{n-1} \frac{P_{i+1} P_{i}}{2} \\
= & n\left(\frac{P_{n+1} P_{n}}{2}\right)-\frac{1}{2}\left(\frac{P_{2 n+1}-2 P_{n+1} P_{n}-1}{4}\right) \\
= & \frac{(4 n+2) P_{n+1} P_{n}-P_{2 n+1}+1}{8}
\end{aligned}
$$


So, the proof is completed. Similarly,

$$
\sum_{k=1}^{n} k Q_{k}^{2}= \begin{cases}\frac{2 n Q_{2 n+1}-Q_{2 n}-4 n-2}{4}, & \text { if } n \text { is odd } \\ \frac{2 n Q_{2 n+1}-Q_{2 n}+4 n+2}{4}, & \text { otherwise }\end{cases}
$$

Corollary $2 P_{n}$ and $Q_{n}$ are $n$th Pell and Pell-Lucas numbers, we have formulas for $\sum_{k=1}^{n} k P_{k}^{2}$ and $\sum_{k=1}^{n} k Q_{k}^{2}$. We can derive a formula for $\sum_{k=1}^{n}(n+1-k) P_{k}^{2}$ and $\sum_{k=1}^{n}(n+1-k) Q_{k}^{2}$.

$$
\begin{gathered}
\sum_{k=1}^{n}(n+1-k) P_{k}^{2}=n P_{1}^{2}+(n-1) P_{2}^{2}+(n-2) P_{3}^{2}+\ldots+1 P_{n}^{2} \\
=(n+1) \sum_{k=1}^{n} P_{k}^{2}-\sum_{k=1}^{n} k P_{k}^{2} \\
=(n+1)\left(\frac{P_{n+1} P_{n}}{2}\right)-\frac{(4 n+2) P_{n+1} P_{n}-P_{2 n+1}+1}{8} \\
=\frac{P_{2 n+1}+2 P_{n+1} P_{n}-1}{8}
\end{gathered}
$$

Using the same technique, we can be show that

$$
\sum_{k=1}^{n}(n+1-k) Q_{k}^{2}= \begin{cases}\frac{Q_{2 n+2}-8 n-10}{4}, & \text { n odd } \\ \frac{Q_{2 n+2}-16 n-14}{4}, & \text { n even }\end{cases}
$$

Theorem 3 Let $A$ be a Hankel-Hessenberg matrix satisfying $a_{i j}=P_{i-j}$, then

$$
\sqrt{\frac{8 n P_{n}^{2}+(4 n-2) P_{n} P_{n-1}-8 P_{n}^{2}-P_{2 n-1}+1}{8 n}} \leq\|A\|_{2} \leq \sqrt{n\left(\frac{P_{n+1} P_{n}}{2}\right)}
$$

where $\|\cdot\|_{2}$ is the spectral norm and $P_{n}$ denotes the $n$th Pell number.

Proof. The matrix $A$ is of the form

$$
\begin{aligned}
& A=\left[\begin{array}{lll}
\operatorname{cccc} P \_0 & P \_1 & P \_n-1
\end{array}\right. \\
& \text { P_1 P_n } \\
& \text { P_n-1 P_n ] }
\end{aligned}
$$

Then we have, 


$$
\begin{gathered}
\|A\|_{E}^{2}=\sum_{k=0}^{n-1}(k+1) P_{k}^{2}+(n-1) P_{n}^{2} \\
=\frac{8 n P_{n}^{2}+(4 n-2) P_{n} P_{n-1}-8 P_{n}^{2}-P_{2 n-1}+1}{8}
\end{gathered}
$$

hence,

$$
\|A\|_{2} \geq \sqrt{\frac{8 n P_{n}^{2}+(4 n-2) P_{n} P_{n-1}-8 P_{n}^{2}-P_{2 n-1}+1}{8 n}}
$$

On the other hand, let the matrices $B$ and $C$ as

$$
\begin{aligned}
& B=\left[\begin{array}{lll}
\operatorname{cccc} 1 & 1 & 1
\end{array}\right. \\
& 11 \\
& 11 \text { ] and } \mathrm{C}=\left[\begin{array}{lll}
\operatorname{cccc} P \_0 & \mathrm{P} \_1 & \mathrm{P} \_\mathrm{n}-1
\end{array}\right. \\
& \text { P_1 P_n } \\
& \text { P_n-1 P_n ] } \\
& \|A\|_{2} \leq \sqrt{n\left(\frac{P_{n+1} P_{n}}{2}\right)}
\end{aligned}
$$

Thus, the proof is completed.

Theorem 4 Let $A$ be a Hankel-Hessenberg matrix satisfying $a_{i j}=Q_{i-j}$, then

\{ where \|\|$_{2}$ is the spectral norm and $Q_{n}$ denotes the $n$th Pell-Lucas number.

Proof. The matrix $A$ is of the form
$A=\left[\begin{array}{lll}\operatorname{cccc} Q \_0 & Q \_1 & Q \_n-1\end{array}\right.$
Q_1 Q_n
Q_n-1 Q_n ]

Then we have,

$$
\begin{aligned}
& \|A\|_{E}^{2}=\sum_{k=0}^{n-1}(k+1) Q_{k}^{2}+(n-1) Q_{n}^{2} \\
& =\{
\end{aligned}
$$

hence,

$$
\|A\|_{2} \geq\{
$$

On the other hand, let the matrices $B$ and $C$ as 
$B=\left[\begin{array}{lll}\operatorname{cccc} 1 & 1 & 1\end{array}\right.$

11

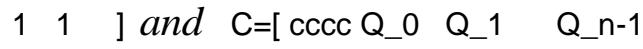

Q_1 Q_n

Q_n-1 Q_n ]

such that $A=B \circ C$. Then

r_1 $(B)=\max$

$\mathrm{i} \_\left|\mathrm{b} \_\mathrm{ij}\right|^{\wedge} 2=\mathrm{j}^{\prime} 1^{\wedge} \mathrm{n}\left|\mathrm{b} \_n \mathrm{j}\right|^{\wedge} 2=\mathrm{n}$ and

C_1 $1(\mathrm{C})=\max$

$\mathrm{j} \_\mathrm{i}\left|\mathrm{c} \_\mathrm{ij}\right|^{\wedge} 2=\_\mathrm{i}=1^{\wedge} \mathrm{n}\left|\mathrm{c} \_\mathrm{in}\right|^{\wedge} 2=\mathrm{i}=1^{\wedge} n Q \_\mathrm{i}{ }^{\wedge} 2=\left\{\mid c r Q \_2 n+1-62\right.$, if $n$ is odd

Q_2n+1-22, otherwise

We have

$$
\begin{aligned}
& \sqrt{n\left(\frac{Q_{2 n+1}-6}{2}\right)}, \quad \text { if } n \text { is odd } \\
& \|A\|_{2} \leq\{ \\
& \sqrt{n\left(\frac{Q_{2 n+1}-2}{2}\right)}, \quad \text { otherwise }
\end{aligned}
$$

Thus, the proof is completed.

Theorem 5 Let $A$ be a Toeplitz-Hessenberg matrix satisfying $a_{i j}=P_{i-j+1}$, then

$$
\sqrt{\frac{P_{2 n+3}-2(2 n+3) P_{n+2} P_{n+1}+8 n^{2}+24 n-1}{8 n}} \leq\|A\|_{2} \leq \sqrt{n\left(\frac{P_{n+2} P_{n+1}-2}{2}\right)}
$$

where $\|\cdot\|_{2}$ is the spectral norm and $P_{n}$ denotes the $n$th Pell number.

Proof. The matrix $A$ is of the form

$A=\left[\begin{array}{lll}\operatorname{cccc} P \_2 & P \_1\end{array}\right.$

P_3

P_1

P_n+1 P_3 P_2 ]

Then we have,

$$
\begin{gathered}
\|A\|_{E}^{2}=\sum_{k=2}^{n+1}(n+2-k) P_{k}^{2}+(n-1) P_{1}^{2} \\
=\frac{P_{2 n+3}-2(2 n+3) P_{n+2} P_{n+1}+8 n^{2}+24 n-1}{8}
\end{gathered}
$$

hence,

$$
\|A\|_{2} \geq \sqrt{\frac{P_{2 n+3}-2(2 n+3) P_{n+2} P_{n+1}+8 n^{2}+24 n-1}{8 n}}
$$

On the other hand, let the matrices $B$ and $C$ as 
$\mathrm{B}=\left[\begin{array}{ll}\operatorname{cccc} 1 & 1\end{array}\right.$

11

1

$\left.\begin{array}{llll}1 & 1 & \ldots & 1\end{array}\right]$ and $\mathrm{C}=\left[\begin{array}{ll}\operatorname{cccc} \mathrm{P} \_2 & \mathrm{P} \_1\end{array}\right.$

P_3 P_2

P_1

P_n+1 P_n ... P_2 ]

such that $A=B \circ C$. Then

r_1(B) $=\max$

$\mathrm{i} \_\mathrm{j}\left|\mathrm{b} \_\mathrm{ij}\right|^{\wedge} 2=\mathrm{j}=1^{\wedge} \mathrm{n}\left|\mathrm{b} \_\mathrm{nj}\right|^{\wedge} 2=\mathrm{n}$ and

C_1 $1(\mathrm{C})=\max$

j_i $\left|c \_i j\right|^{\wedge} 2=\_i=1^{\wedge} n\left|c \_i n\right|^{\wedge} 2=\_i=2^{\wedge} n+1 P \_i \wedge 2=P \_n+2 P \_n+1-22$

We have

$$
\|A\|_{2} \leq \sqrt{n\left(\frac{P_{n+2} P_{n+1}-2}{2}\right)}
$$

Thus, the proof is completed.

Theorem 6 Let $A$ be a Toeplitz-Hessenberg matrix satisfying $a_{i j}=Q_{i-j+1}$, then

$$
\begin{cases}\sqrt{\frac{Q_{2 n+2}+8 n-26}{4 n}} & \leq\|A\|_{2} \leq \sqrt{n\left(\frac{Q_{2 n+1}-6}{2}\right)}, \quad \text { if } n \text { is odd } \\ \sqrt{\frac{Q_{2 n+2}-30}{4 n}} & \leq\|A\|_{2} \leq \sqrt{n\left(\frac{Q_{2 n+1}-2}{2}\right)}, \quad \text { otherwise }\end{cases}
$$

where \|\|$_{2}$ is the spectral norm and $Q_{n}$ denotes the $n$th Pell-Lucas number.

Proof. The matrix $A$ is of the form

$\mathrm{A}=\left[\begin{array}{ll}\operatorname{cccc} Q_{-} 1 & \mathrm{Q} \_0\end{array}\right.$

Q_2

Q_0

Q_n Q_2 Q_1]

Then we have,

$$
\begin{aligned}
& \|A\|_{E}^{2}=\sum_{k=1}^{n}(n+1-k) Q_{k}^{2}+(n-1) Q_{0}^{2} \\
& \frac{Q_{2 n+2}+8 n-26}{4} \text {, if } n \text { is odd } \\
& =\{ \\
& \frac{Q_{2 n+2}-30}{4}, \quad \text { otherwise }
\end{aligned}
$$

hence, 


$$
\|A\|_{2} \geq \begin{cases}\sqrt{\frac{Q_{2 n+2}+8 n-26}{4 n}}, & \text { if } n \text { is odd } \\ \sqrt{\frac{Q_{2 n+2}-30}{4 n}}, & \text { otherwise }\end{cases}
$$

On the other hand, let the matrices $B$ and $C$ as

$$
\begin{aligned}
& \mathrm{B}=[\operatorname{cccc} 1 \quad 1 \\
& 11 \\
& \left.\begin{array}{llll}
1 & 1 & \ldots & 1
\end{array}\right] \text { and } \quad \mathrm{C}=\left[\begin{array}{lll}
\operatorname{cccc} Q & 1 & \mathrm{Q}
\end{array}\right. \\
& \text { Q_2 Q_1 } \\
& \text { Q_0 } \\
& \text { Q_n Q_n-1 ... Q_1 ] }
\end{aligned}
$$

We have

$$
\begin{aligned}
& \sqrt{n\left(\frac{Q_{2 n+1}-6}{2}\right)}, \quad \text { if } n \text { is odd } \\
& \|A\|_{2} \leq\{ \\
& \sqrt{n\left(\frac{Q_{2 n+1}-2}{2}\right)}, \quad \text { otherwise }
\end{aligned}
$$

Thus, the proof is completed.

\section{References}

[1] M. Akbulak, D. Bozkurt, On The Norms Of Toeplitz Matrices Involving Fibonacci And Lucas Numbers, Hacettepe Journal of Mathematics and Statistics, 37(2), 89-95, (2008).

[2] M. Bah S i, S. Solak, On the norms of r-circulant matrices with the hyper-Fibonacci and Lucas numbers, Journal of Mathematical Inequalities, 8(4), 693-705, (2014).

[3] M. Merca, A Note On The Determinant Of a Toeplitz-Hessenberg Matrix, Special Matrices, DOI: 10.2478/spma-20130003, (2013).

[4] R. A. Horn, C. R. Johnson, Topics in Matrix Analysis, Cambridge University Press, New York, (1991).

[5] H. Civciv, R. Türkmen, Notes on norms of circulant matrices with lucas numbers, Int. Jour. of Inf. and Systems Sciences, 4(1), 142-147, (2008).

[6] R. Türkmen, H. Gökba S , On the Spectral Norm of $r$-Circulant Matrices with Pell and Pell-Lucas numbers, Journal of Inequalities and Applications, 2016:65, DOI: 10.1186/s13660-016-0997-0, (2016).

[7] S. Shen, J. Cen, On the spectral norms of r-circulant matrices with the k-Fibonacci and k-Lucas numbers, Int. J. Contemp. Math. Sciences, 5(12), 569-578, (2010).

[8] T. Koshy, Fibonacci and Lucas Numbers with Applications, A Wiley-Interscience Publication, Canada, (2001).

[9] T. Koshy, Pell and Pell-Lucas Numbers with Applications, Springer, New York, (2014). 